\title{
Higher Order Photon Transitions in H-like and
}

\section{He-like Ions}

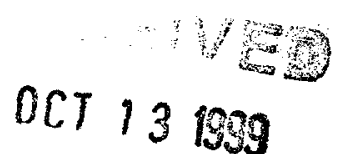

R. W. Dunford ${ }^{1}$, E. P. Kanter ${ }^{1}$, H. W. Schäffer ${ }^{2}$, P. H. Mokler ${ }^{2}$, H. G. Berry ${ }^{3}$, A. E. Livingston ${ }^{3}$, S. Cheng ${ }^{4}$, L. J. Curtis ${ }^{4}$

1 Physics Division, Argonne National Laboratory, Argonne Il 60439, USA

2 Gesellschaft für Schwerionenforschung mbH, 64291 Darmstadt, Germany

${ }^{3}$ Department of Physics, University of Notre Dame, Notre Dame, Indiana 46556, USA

${ }^{4}$ Department of Physics and Astronomy, University of Toledo, Toledo, Ohio 43606, USA

PACS numbers: 32.70.Fw, 31.30.Jv, 31.10.+z 


\section{DISCLAIMER}

This report was prepared as an account of work sponsored by an agency of the United States Government. Neither the United States Government nor any agency thereof, nor any of their employees, make any warranty, express or implied, or assumes any legal liability or responsibility for the accuracy, completeness, or usefulness of any information, apparatus, product, or process disclosed, or represents that its use would not infringe privately owned rights. Reference herein to any specific commercial product, process, or service by trade name, trademark, manufacturer, or otherwise does not necessarily constitute or imply its endorsement, recommendation, or favoring by the United States Government or any agency thereof. The views and opinions of authors expressed herein do not necessarily state or reflect those of the United States Government or any agency thereof. 


\section{DISCLAIMER}

Portions of this document may be illegible in electronic image products. Images are produced from the best available original document. 


\begin{abstract}
Higher order photon transitions such as $M 1, M 2$ and two-photon decay are conveniently studied using highly-charged few electron ions. Here we discuss two examples from recent experiments which were done using the ATLAS facility at Argonne National Laboratory. The first is a test of Relativistic Quantum Mechanics involving a precision measurement of the spectral shape of the two-photon decay of the $1 s 2 s^{1} S_{0}$ state in He-like nickel and the second is a test of the theory of damping in quantum mechanics involving observation of $E 1-M 1$ interference in the electric field quenching of metastable H-like ions.
\end{abstract}

\title{
1 Introduction
}

The study of atomic transition probabilities is an important part of research in atomic structure. Such studies compliment work in measurement of transition energies because they provide more sensitivity to atomic wavefunctions and address issues such as the handling of the negative energy states $[1,2,3]$.

Few-electron, highly charged ions provide a good testing ground for the interplay of relativistic effects and interelectron interactions. Lifetime measurements can be difficult in this regime, however, because the states are very 
short lived. One solution is to study forbidden transitions from metastable states. Such states become shorter-lived as $Z$ is increased and come into the regime of lifetimes that are convenient to study experimentally.

In this paper, we describe two recent experiments involving higher order transitions which have been done at the ATLAS heavy ion accelerator at Argonne National Laboratory. The first is a study of the spectral shape of the two-photon decay of the $1 \mathrm{~s} 2 \mathrm{~s}{ }^{1} S_{0}$ level in heliumlike nickel which provides a test of relativistic quantum mechanics in the intermediate $Z$ regime. The second is a study of the angular distribution of the radiation resulting from the electric field quenching of the $2 s$ state of H-like Ar. This experiment provides a test of the theory of damping in quantum mechanics.

\section{Two-Photon Decay}

Studies of two-photon decay are important as they test our understanding of the entire structure of an atom, since the amplitude includes contributions from all discrete and continuum states. Most of the studies of two-photon decay have relied on measurement of the lifetimes of two-photon emitting states $[4,5]$. For high- $Z$ ions the lifetimes become too short to measure 
accurately, however, so we have undertaken a study of the shape of the continuum from two-photon decay. Such measurements can be done for any ion and they provide information beyond what can be obtained from lifetime measurements which measure the transition probability integrated over the photon energies.

The spectral distribution from two-photon decay of the $1 s 2 s^{1} S_{0}$ in He-like nickel was measured in a recent experiment at ATLAS [6]. In this experiment, we were able to overcome a limitation in an earlier measurement [7] of the spectral shape in He-like $\mathrm{Kr}$ which was due to uncertainty in the energydependent efficiencies of the detectors. In the nickel experiment, two-photon decay in both $\mathrm{H}$-like and He-like ions was measured in the same experiment, switching between the two species periodically. Since the continuum shape in the two-photon decay of the $2 s^{2} S_{1 / 2}$ level in H-like nickel is known precisely, these data served as a calibration of the spectral efficiency of the detection system which then allowed a precision determination of the spectral distribution in the He-like two-photon transition.

In the experiment $\mathrm{Ni}^{26+}$ or $\mathrm{Ni}^{28+}$ ions were directed to the target chamber. With $N i^{26+}$ incident on target, the $1 s 2 s^{1} S_{0}$ level in He-like $\mathrm{Ni}$ was formed via excitation, whereas, with $N i^{28+}$ incident on the target the $2 s$ 
${ }^{2} S_{1 / 2}$ level in $\mathrm{H}$-like $\mathrm{Ni}$ was formed via capture. Photon coincidences associated with the decays were observed with an array of three $\mathrm{Si}(\mathrm{Li}) \mathrm{x}$-ray detectors.

A Monte Carlo code [8] was developed to simulate the actual shapes of the $\mathrm{x}$-ray continua on the basis of present theories. By comparing the results of the Monte-Carlo simulation for H-like nickel with our data for the twophoton decay of the $2 s^{2} S_{1 / 2}$ state, we were able to constrain the simulation and obtain a model-independent determination of the shape of the continuum distribution in He-like nickel. In Fig. 1 we compare our results (points with error bars) with various calculations. The data show excellent agreement with both Johnson's relativistic calculations [9] and Drake's nonrelativistic calculations $[10,11]$ for nickel. These results provide a precise measurement of the spectral distribution for two-photon decay at intermediate $Z$. The next step is to compare this result with a measurement in He-like gold $(Z=79)$ where the relativistic corrections are more important. The first data from this part of our program has already been obtained in an experiment done at GSI, Darmstadt. See the contribution by Schäffer et al. in this volume. 


\section{Damping Asymmetry}

The metastable $2 s^{2} S_{1 / 2}$ state in one-electron ions decays to the ground state either by two-photon decay or by single-photon $M 1$ decay. If an external electric field $\mathrm{E}$ is applied to the ion there is, in addition, a Stark-induced $E 1$ amplitude. Interference between the $E 1$ and $M 1$ amplitudes leads to an asymmetry in the angular distribution proportional to the invariant $\mathbf{k} \cdot \mathbf{E}$ which is of interest because it appears to violate time reversal $(T)$ invariance. It has been shown however, that even in a $T$-conserving theory this term can be nonvanishing if damping is taken into account $[12,13]$. We have made the first observation of this $E 1-M 1$ damping-interference [14]. Our results provide a test of damping in quantum mechanics and can also be interpreted as a measurement of the Lamb shift in H-like Ar. $E 1-M 1$ interference in the quenching of the $2 s^{2} S_{1 / 2}$ level in polarized $\mathrm{He}^{+}$ions has been measured by van Wijngaarden and Drake $[15,16,17,18]$. A recent review of anisotropy measurements in H-like atoms is given in Ref. [15].

In the experiment, ions formed in the metastable $2 s^{2} S_{1 / 2}$ level in $\mathrm{H}$ like Ar proceeded to the center of a chamber where their decay radiation was monitored by two $\mathrm{Si}(\mathrm{Li}) \mathrm{X}$-ray detectors located on opposite sides of the beam. A magnetic field $B$ was applied perpendicular to both the beam 
velocity and the detector axis. Because of their motion through this field, the ions experienced an electric field $\mathbf{E}=\gamma \frac{\mathbf{v}}{c} \times \mathbf{B}$ in their rest frame. The electric field was perpendicular to the beam velocity and parallel to the direction of the detected photons. In Fig. 2 we show typical spectra taken with one of the $\mathrm{Si}(\mathrm{Li})$ detectors at several values of magnetic field. The peak near $3 \mathrm{keV}$ is a blend of Lyman- $\alpha$ and $\mathrm{K} \alpha$ lines from H-like and He-like ions. Because of the Stark-induced $E 1$ transitions, the H-like peak becomes more intense as the magnetic field is increased. The part of the $\mathbf{E} \cdot \mathbf{k}$ asymmetry that changes sign when the magnetic field is reversed is called $r_{2}$. Data were taken at a number of different magnetic field strengths and fit to a straight line determining the slope:

$$
r_{2}=(-0.01666 \pm 0.00041) \times B(k G)
$$

which agrees with the expected value based on the theoretical calculation[12, 13]. If we interpret these data as a determination of the Lamb shift $S$ we find $S_{\text {exp }}=38.6(1.4) T H z$ in agreement with the theoretical result [19] of $S_{t h}=$ 38.2445 THz. Our result is higher than but in substantial agreement with the experimental result of Gould and Marrus [20] $S_{G M}=37.89(0.38) T h z$.

In the future, the apparatus developed for this program will be used to measure the polarization of metastable ions which have been created in an 
array of tilted foils [21]. Polarization would be measured by observing the angular distribution of photons arising from the quenching of the metastable ions in an electric field.

\section{Acknowledgments}

This work was supported by the US Department of Energy, Office of Basic Energy Sciences, Division of Chemical Sciences, and by a NATO travel grant. 


\section{References}

[1] W. R. Johnson, D. R. Plante, and J. Sapirstein, in Advances in Atomic, Molecular and Optical Physics, edited by B. Bederson and H. Walther (Academic Press, San Diego, 1995), Vol. 35, p. 255.

[2] P. Indelicato, Phys. Rev. Lett. 77, 3323 (1996).

[3] J. J. Sakurai, Advanced Quantum Mechanics, (Addison-Wesley, Reading, Massachusetts, 1967).

[4] R. W. Dunford, H. G. Berry, D. A. Church, M. Hass, C. J. Liu, M. L. A. Raphaelian, B. J. Zabransky, L. J. Curtis, and A. E. Livingston, Phys. Rev. A 48, 2729 (1993).

[5] A. Simionovici, B. B. Birkett, J. P. Briand, P. Charles, D. D. Dietrich, K. Finlayson, P. Indelicato, D. Liesen, and R. Marrus, Phys. Rev. A 48, 1695 (1993).

[6] H. W. Schäffer, R. W. Dunford, E. P. Kanter, S. Cheng, L. J. Curtis, A. E. Lịvingston, and P. H. Mokler, in preparation. 
[7] R. Ali, I. Ahmad, R. W. Dunford, D. S. Gemmell, M. Jung, E. P. Kanter, P. H. Mokler, H. G. Berry, A. E. Livingston, S. Cheng, and L. J. Curtis, Phys. Rev. A 55, 994 (1997).

[8] Code available at ftp://anlphy.phy.anl.gov/mc2p.

[9] A. Derevianko and W. R. Johnson, Phys. Rev. A 56, 1288 (1997).

[10] G. W. F. Drake, unpublished report, University of Windsor, 1988.

[11] G. W. F. Drake, Phys. Rev. A 34, 2871 (1986).

[12] P. J. Mohr, Phys. Rev. Lett. 40, 854 (1978).

[13] M. Hillery and P. J. Mohr, Phys. Rev. A 21, 24 (1980).

[14] R. W. Dunford, D. S. Gemmell, M. Jung, E. P. Kanter, H. G. Berry, A. E. Livingston, S. Cheng, and L. J. Curtis, Phys. Rev. Lett. 79, 3359 (1997).

[15] G. W. F. Drake and A. v. Wijngaarden, in Selected Topics on Electron Physics, edited by D. M. Campbell and H. Kleinpoppen (Plenum, New York, 1996), p. 411.

[16] G. W. F. Drake, in The Spectrum of Atomic Hydrogen: Advances, edited by G. W. Series (World Scientific, Singapore, 1988), p. 137. 
[17] A. van Wijngaarden and G. W. F. Drake, Phys. Rev. A 25, 400 (1982).

[18] A. van Wijngaarden, J. Patel, and G. W. F. Drake, Phys. Rev. A 33, $312(1986)$

[19] P. J. Mohr, in Atomic Molecular, and Optical Physics Handbook, edited by G. W. F. Drake (AIP, Woodbury, New York, 1996), p. 341.

[20] H. Gould and R. Marrus, Phys. Rev. A 28, 2001 (1983).

[21] R. W. Dunford, J. Phys. B 30, 523 (1997). 


\section{Figure Captions}

Fig. 1. Rate for two photon decay as a function of the photon energy (in units of the fraction of the transition energy). The data for He-like nickel are compared with various theoretical results. The data and theory have been divided by a factor $\omega_{1}\left(\omega_{0}-\omega_{1}\right)$ where $\omega_{1}$ is the photon energy and $\omega_{0}$ is the transition energy.

Fig. 2. Spectrum measured with detector $B$ for three different magnetic field settings. 


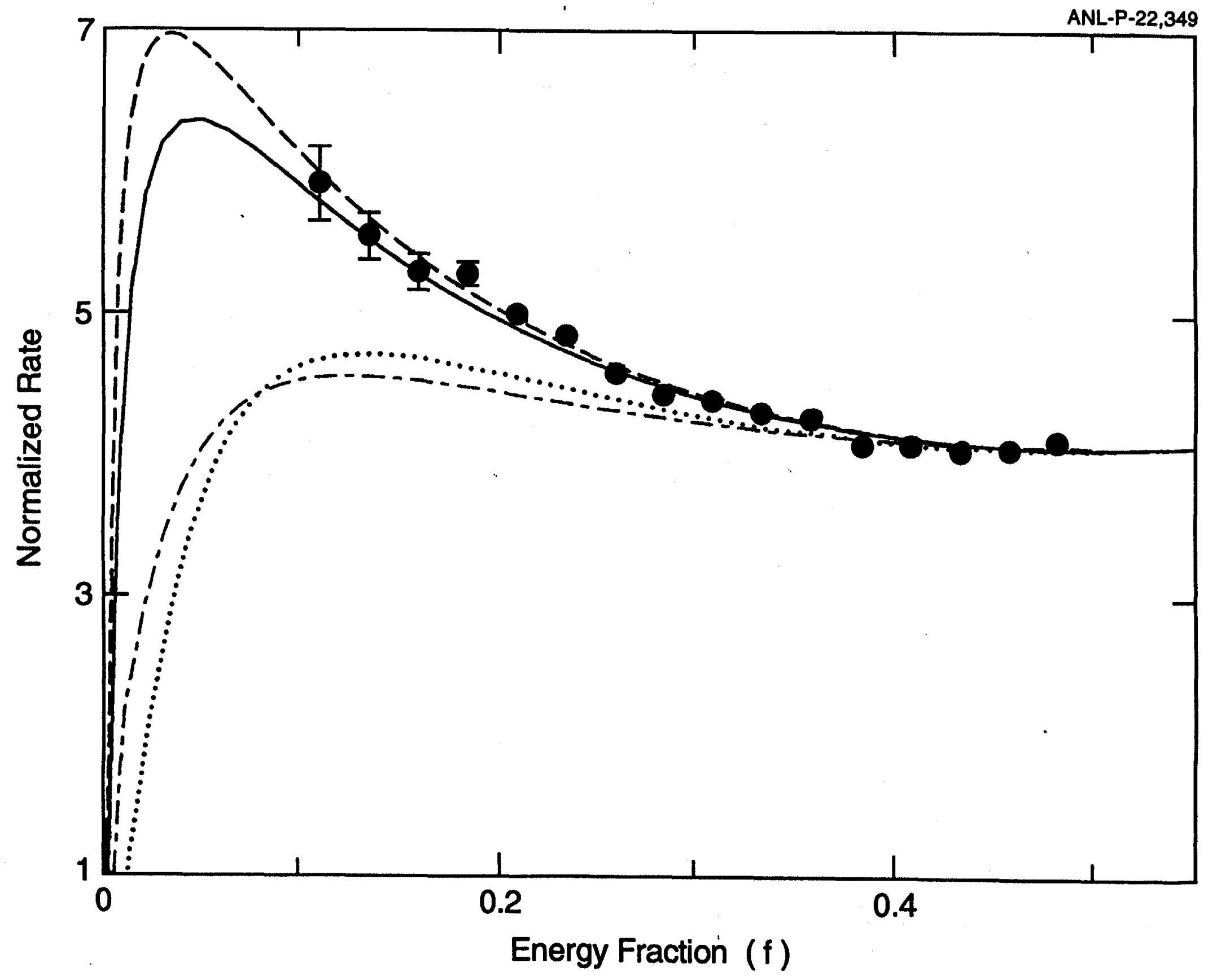




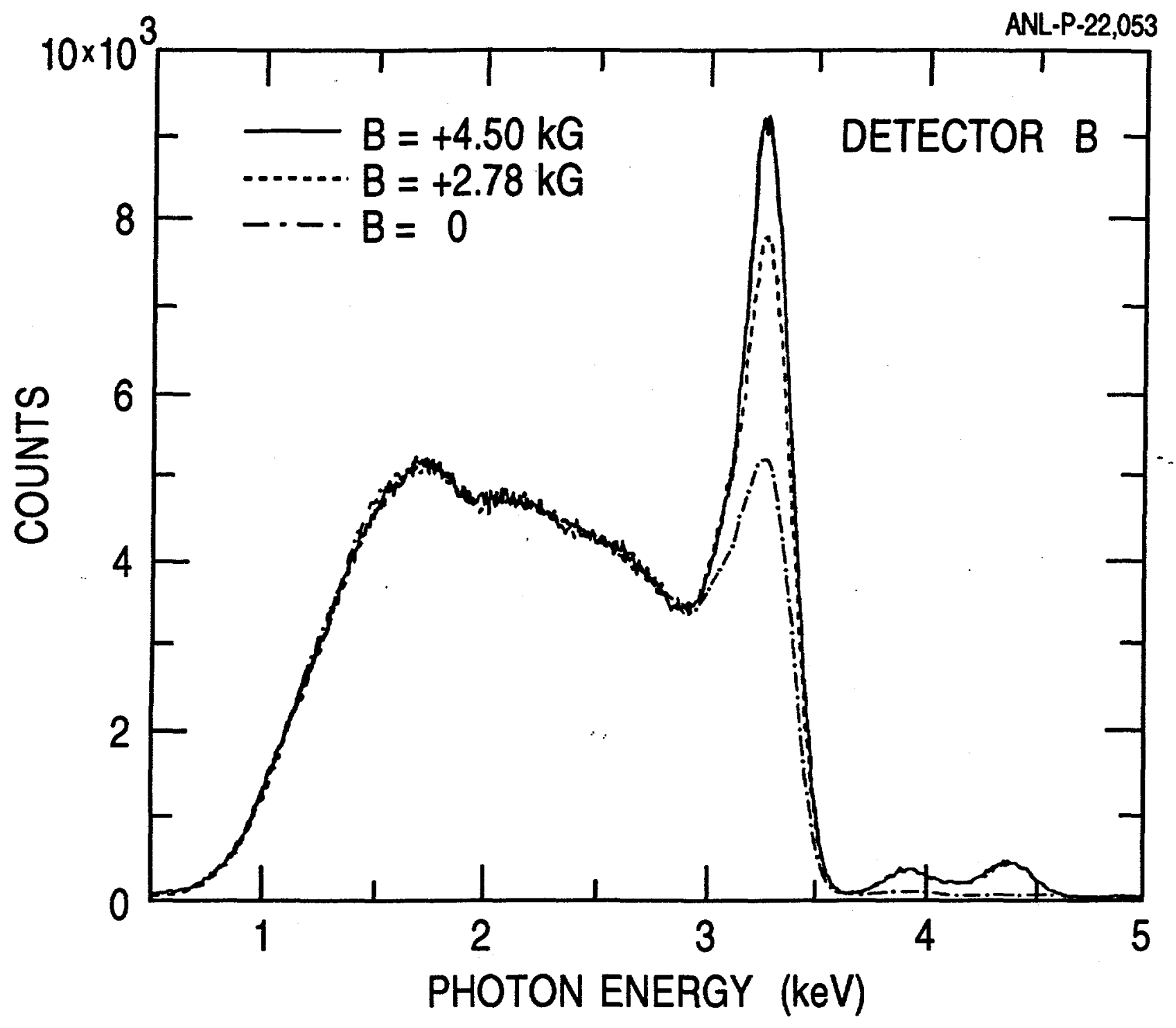

FIG.2 